\title{
Single-file Diffusion of Atomic and Colloidal Systems: Asymptotic Laws
}

\author{
Markus Kollmann* \\ Institut für Festkörperforschung, Teilinstitut Weiche Materie, Forschungszentrum Jülich, D-52425 Jülich, Germany
}

(Received 22 November 2002; published 9 May 2003)

\begin{abstract}
We present a general derivation of the non-Fickian behavior for the self-diffusion of identically interacting particle systems with excluded mutual passage. We show that the conditional probability distribution of finding a particle at position $x_{t}$ after time $t$, when the particle was located at $x_{0}$ at $t=0$, follows a Gaussian distribution in the long-time limit, with variance $2 W(t) \sim t^{1 / 2}$ for overdamped systems and with variance $2 W(t) \sim t$ for classical systems. The asymptotic behavior of the meansquared displacement, $W(t)$, is shown to be independent of the nature of interactions for homogeneous systems in the fluid state. Moreover, the long-time behavior of self-diffusion is determined by shorttime and large-scale collective density fluctuations.
\end{abstract}

DOI: 10.1103/PhysRevLett.90.180602

Single-file diffusion refers to the motion of particles in narrow pores so that the individual particles are unable to pass each other and the sequence of particle labels remains the same over time. As the mutual passage is excluded, the motion of individual particles requires the collective motion of many other particles in the same direction. This restriction leads to an anomalous behavior of the self-diffusion for overdamped systems in the longtime limit, which has been subject to long-standing theoretical investigations [1-3]. For classical systems normal diffusive behavior has been found for the self-diffusion function [4-7]. So far, rigorous results have been derived for the long-time behavior of the conditional probability density function (PDF), $P\left(y_{t}, t \mid y_{0}, 0\right)$, of finding a particle at position $y_{t}$ at time $t$, being located at $y_{0}$ at time $t=0$, only for one-dimensional hard-rod systems using various analytical methods $[1,2,4,8]$. For these systems it has been shown that $P\left(y_{t}, t \mid y_{0}, 0\right)$ is Gaussian with variance $2 W(t)$ for asymptotically large times. The function $W(t)$ is the mean-squared displacement (MSD) given by $W(t)=$ $A_{o} t^{1 / 2}$ and $W(t)=A_{c} t$ for overdamped and classical systems, respectively. The constants $A_{o}$ and $A_{c}$ depend on thermodynamic properties and short-time transport coefficients of the particular systems. Although these results have been derived for hard-rod systems only, it is generally believed that in systems with prohibited exchange of particle labels the MSD scales like $W(t) \sim$ $t^{1 / 2}$ in overdamped systems [9] and like $W(t) \sim t$ in classical systems.

Experimental evidence confirming the anomalous diffusive behavior has been found only recently in atomic $[10,11]$ and colloidal systems [12]. The experimental results support the conjectured scaling behavior of the MSD as given above [9]. The single-filing condition is hard to fulfill experimentally for atomic systems, whereas for micrometer-sized colloidal particles narrow channels can easily be generated [12]. While atoms follow ballistic motion, colloidal particles follow overdamped motion due to the presence of the solvent. Moreover, in colloidal systems there exist additional correlations due to solvent
PACS numbers: 05.40.Jc, 82.70.-y

mediated hydrodynamic interactions (HI) [13]. Nevertheless, the experiments, using magnetic colloids with long-range dipole-dipole pair interactions, show clearly the same long-time scaling of the MSD as predicted for hard-rod systems with HI neglected.

So far a general theory for the self-diffusion in homogeneous single-filing atomic and Brownian systems was still lacking and will be given in this Letter. We show that the general conjectured scaling of the MSD for singlefiling systems [9] is indeed true and derive asymptotic laws for the single-file diffusion of colloidal and atomic particle systems with arbitrary interaction potential, under the condition that the correlation length between the particles is of finite range and the particles interact identically. We prove that in the limit of times, much larger than the time needed for a particle to diffuse the mean particle distance, the conditional PDF $P\left(y_{t}, t \mid y_{0}, 0\right)$ is normal distributed with variance $2 W(t)$. Moreover, we include HI in the case of colloidal systems and give explicit expressions for the prefactor $A$ in the expression for the MSD. We show that this prefactor is determined by the compressibility of the system and the short-time collective diffusion coefficient and not by the shorttime self-diffusion coefficent which could have been expected from previous work [8].

Overdamped systems. - We consider a one-dimensional homogeneous system of $N$ identically interacting Brownian particles in equilibrium. The system size is of length $L$ and assumed to be macroscopically large. The total number density of the particles in the system is denoted by $\bar{\rho}=N / L$ and the mean particle distance is given by $a=\bar{\rho}^{-1}$. The interaction potential is chosen to be infinite at overlap of any two particle centers. Consequently, the particles are unable to pass each other. In the overdamped limit the time evolution of the particle trajectories follow the stochastic equations [14]

$$
\frac{\partial y_{i}(t)}{\partial t}=\hat{\Omega}_{B}\left[y^{N}(t)\right] y_{i}(t)+\eta_{i}(t)
$$

with $\hat{\Omega}_{B}$ the so-called backward Smoluchowski operator 
$[13,15]$ given by

$$
\hat{\Omega}_{B}\left[y^{N}\right]=\sum_{i, j=1}^{N}\left[\frac{\partial}{\partial y_{j}}-\frac{\partial U\left(y^{N}\right)}{\partial y_{j}}\right] D_{i j}\left(y^{N}\right) \frac{\partial}{\partial y_{i}} .
$$

Here, $y_{i}(t)$ is the position of particle $i$ at time $t, D_{i j}\left(y^{N}\right)$ is the diffusivity tensor, accounting for solvent mediated hydrodynamic interactions, and $U\left(y^{N}\right)$ is the interaction potential among the particles in the system, given in units of $k_{B} T$. We also make use of the supervector notation $y^{N}(t)=\left\{y_{1}(t), \ldots, y_{N}(t)\right\}$. The random noise $\eta_{i}(t)$ is normal distributed with mean zero, and the second moment is given by $\left\langle\eta_{i}(t) \eta_{j}\left(t^{\prime}\right)\right\rangle=2 D_{i j}\left(y^{N}\right) \delta\left(t-t^{\prime}\right)$. We emphasize that the stochastic equations have to be interpreted in the Ito sense [16]. The basic quantity we want to calculate is the MSD, $W(t)$, given by

$$
W(t)=\frac{1}{2}\left\langle\left[y_{i}(t)-y_{i}(0)\right]^{2}\right\rangle
$$

for asymptotically large times $t$. The index $i$ is arbitrary but chosen such that the particle under consideration is located sufficiently far from the walls for the timeinterval considered. The brackets, \langle\rangle , indicate an average with respect to the joint probability density function, $P\left(y_{t}^{N}, t \mid y_{0}^{N}, 0\right) P_{\text {eq }}\left(y_{0}^{N}\right)$, of finding the system at position $y_{t}^{N}$ at time $t$ and at position $y_{0}^{N}$ at time $t=0$ in configuration space. Here, $P_{\mathrm{eq}}\left(y^{N}\right)=\exp \left[-U\left(y^{N}\right)\right] / Z_{N}$ is the Boltzmann distribution and $Z_{N}$ the configurational integral. The conditional PDF, $P\left(y_{t}^{N}, t \mid y_{0}^{N}, 0\right)$, satisfies the differential equation

$$
\partial_{t} P\left(y_{t}^{N}, t \mid y_{0}^{N}, 0\right)=\hat{\Omega}\left[y_{t}^{N}\right] P\left(y_{t}^{N}, t \mid y_{0}^{N}, 0\right)
$$

with initial condition $\lim _{t \rightarrow 0^{+}} P\left(y_{t}^{N}, t \mid y_{0}^{N}, 0\right)=\delta\left(y_{t}^{N}-y_{0}^{N}\right)$. In Eq. (4), the operator $\hat{\Omega}$ is the ajoint operator of $\hat{\Omega}_{B}$. As we assume the system to be ergodic and in equilibrium, the conditional PDF $P\left(y_{t}^{N}, t \mid y_{0}^{N}, 0\right)$ will approach the Boltzmann distribution, $P_{\text {eq }}\left(y^{N}\right)$, in the long-time limit. Hence, $\lim _{t \rightarrow \infty} W(t)$ is of quadratic order in the system size. As $W(t)$ is a monotonically increasing function of time, the major contribution to the MSD at large times is given by particle trajectories whose end to end distances are much larger than the mean particle distance, $a$. In the following we look for an expression for the MSD in the limit $t \gg \tau=a^{2} / D_{S}$, with $D_{S}$ the short-time selfdiffusion coefficient of a free diffusing particle.

To simplify the calculation for $W(t)$ we introduce an auxiliary stochastic function $x(t)$, defined by $y_{i}(t)<$ $x(t)<y_{i+1}(t)$, and the microscopic density $\hat{\rho}(y, t)=$ $\sum_{i}^{N} \delta\left[y-y_{i}(t)\right]$. By definition we have $W(t)=\tilde{W}(t)+$ $\mathcal{O}(a)$ with $\tilde{W}(t)=1 / 2\left\langle[x(t)-x(0)]^{2}\right\rangle$. Consequently, the long-time behavior of $W(t)$ and $\tilde{W}(t)$ for infinite system size are related by

$$
\lim _{t \rightarrow \infty} \lim _{L \rightarrow \infty} \frac{\tilde{W}(t)}{W(t)}=1 .
$$

In order to calculate $\tilde{W}(t)$ we have to derive an explicit expression for the conditional PDF, $\tilde{P}\left(x_{t}, t \mid x_{0}, 0\right)$, of finding trajectory of $x(t)$ at position $x_{t}$ at time $t$ when it was located at $x_{0}$ at time $t=0$.

To this end, it is useful to introduce another stochastic variable, $y_{i+M}(t)<z(t)<y_{i+M+1}(t)$, and take $M$ so large such that $\left\langle x\left(t^{\prime}\right) z\left(t^{\prime \prime}\right)\right\rangle=0$ for all times $t^{\prime}, t^{\prime \prime} \leq t$. By homogeneity of the systems, $x(t)$ and $z(t)$ are stochastically equivalent variables. Now, the conditional PDF, $\tilde{P}_{r}\left(x_{t}-\right.$ $\left.z_{t}, t \mid x_{0}-z_{0}, 0\right)$, to find the two trajectories, $x(t)$ and $z(t)$, separated a distance $x_{t}-z_{t}$ at time $t$ when they were separated a distance $x_{0}-z_{0}$ at time $t=0$ is then given by

$$
\tilde{P}_{r}\left(\Delta x_{t}-\Delta z_{t}, t\right)=\int_{L} \tilde{P}\left(\Delta x_{t}+a, t\right) \tilde{P}\left(\Delta z_{t}+a, t\right) d a .
$$

Here, $\Delta x_{t}=x_{t}-x_{0}, \Delta z_{t}=z_{t}-z_{0}$, and we employed translationary invariance and stationarity for the conditional PDF, $\tilde{P}\left(\Delta x_{t}, t\right)=\tilde{P}\left(x_{t}, t \mid x_{0}, 0\right)$. From the knowledge of $\tilde{P}_{r}\left(\Delta x_{t}-\Delta z_{t}, t\right)$, one can determine uniquely the conditional PDF $\tilde{P}\left(\Delta x_{t}, t\right)$ by making use of the convolution theorem.

In the following we determine the function $\tilde{P}_{r}\left(\Delta x_{t}-\right.$ $\left.\Delta z_{t}, t\right)$ from the stochastic properties of the microscopic density, $\hat{\rho}(y, t)$. To this end, we introduce the functional

$$
h\left(x_{t}, z_{t} \mid x_{0}, z_{0}\right):=\int_{z_{t}}^{x_{t}} \hat{\boldsymbol{\rho}}(y, t) d y-\int_{z_{0}}^{x_{0}} \hat{\boldsymbol{\rho}}\left(y^{\prime}, 0\right) d y^{\prime},
$$

together with the general condition

$$
\lim _{t \rightarrow \infty} \lim _{N, L \rightarrow \infty} \operatorname{Prob}\left\{(x(t)-x(0))^{2}>\tilde{W}(t)^{1+\epsilon}\right\}=0,
$$

with $\epsilon>0$ arbitrary small. Consequently, we get by definition of $x(t), z(t)$

$$
h[x(t), z(t) \mid x(0), z(0)]=0 .
$$

The conditional PDF, $\tilde{P}_{r}\left(\Delta x_{t}-\Delta z_{t}, t\right)$, is then given in the long-time regime by

$$
\begin{aligned}
\tilde{P}_{r}\left(\Delta x_{t}-\Delta z_{t}, t\right)=\lim _{l \rightarrow \infty} \lim _{t \gg \tau, L \rightarrow \infty}\langle & \delta_{l}\left[h\left(x_{t}, z_{t} \mid x_{0}, z_{0}\right)\right] \\
& \left.\times\left|\frac{\partial h\left(x_{t}, z_{t} \mid x_{0}, z_{0}\right)}{\partial\left(x_{t}-z_{t}\right)}\right|\right\rangle .
\end{aligned}
$$

Here, $t \gg \tau$ indicates the asymptotic limit for large times and $\delta[z]=\lim _{l \rightarrow \infty} \delta_{l}[z]$ is the delta distribution in the Dirac sense. The reason why we take the asymptotic limit $t \gg \tau$ before taking the limit $l \rightarrow \infty$ is because the conditional probability density, $\tilde{P}_{r}\left(\Delta x_{t}-\Delta z_{t}, t\right)$, cannot be normalized in a simple way if $l \rightarrow \infty$ is taken first. By definition of $x(t)$ there corresponds an infinite set of realizations of $\hat{\rho}(y, t)$ to one given realization of $x(t)$ which leads to divergent contributions for $\left\langle\delta\left[h\left(x_{t}, z_{t} \mid x_{0}, z_{0}\right)\right]\right\rangle$. Performing the asymptotic analysis for long times first, we expect to lose all information on scales of the mean particle distance, and hence there is 
some chance that the same form of normalization is valid, as used in Eq. (10) for a finite set of realizations of $\hat{\rho}(y, t)$ corresponding to one realization of $x(t)$. We emphasize that the right-hand sides of Eqs. (6) and (10) are stochastical equivalent only in the long-time limit, as in Eq. (10) we get additional fluctuations because in Eq. (7) there enters no information that the amount of trajectories of

$x(t)$ traveling infinitely far during a finite time is of measure zero. These additional fluctuations do not contribute in the long-time limit, as can be shown by inserting in Eq. (13) any value between the upper bound, as given in Eq. (8), and the lower bound, $x(t)-x(0)=0$, for $x(t)$.

The conditional PDF for the relative motion, $\tilde{P}_{r}\left(\Delta x_{t}-\right.$ $\left.\Delta x_{0}, t\right)$, can be rewritten in integral form

$$
\tilde{P}_{r}\left(\Delta x_{t}-\Delta z_{t}, t\right)=\lim _{l \rightarrow \infty} \lim _{t \gg \tau_{I}} \int\left|\frac{1}{2 \pi i \xi} \frac{\partial}{\partial\left(x_{t}-z_{t}\right)} \exp \left[\sum_{n=1}^{\infty} \frac{(i \xi)^{n}}{n !} \kappa^{(n)}-\xi^{2} /(2 l)\right] d \xi\right|
$$

with $\kappa^{(n)}$ the $n$th cumulant of $h\left(x_{t}, z_{t} \mid x_{0}, z_{0}\right)$. In the following we show that in the limit $t \gg \tau$ only the first two cumulants contribute to $\tilde{P}_{r}\left(\Delta x_{t}-\Delta z_{t}, t\right)$. The first cumulant reads simply

$$
\boldsymbol{\kappa}^{(1)}=\left\langle h\left(x_{t}, z_{t} \mid x_{0}, z_{0}\right)\right\rangle=\bar{\rho}\left[\left(x_{t}-z_{t}\right)-\left(x_{0}-z_{0}\right)\right] .
$$

The second cumulant can be expressed as

$$
\begin{aligned}
\kappa^{2}=\left\langle h\left(x_{t}, z_{t} \mid x_{0}, z_{0}\right)^{2}\right\rangle- & \left\langle h\left(x_{t}, z_{t} \mid x_{0}, z_{0}\right)\right\rangle^{2} \\
=\frac{\bar{\rho}}{\pi} \int \frac{1}{q^{2}}\{2 S(q, 0)- & \left(\exp \left[-i q\left(x_{t}-x_{0}\right)\right]\right. \\
& \left.\left.+\exp \left[i q\left(z_{t}-z_{0}\right)\right]\right) S(q, t)\right\} d q .
\end{aligned}
$$

Here, we introduced the dynamic structure factor defined by $S(q, t)=\left\langle\delta \hat{\rho}(q, t) \delta \hat{\rho}^{*}(q, 0)\right\rangle / N$, with $\delta \hat{\rho}(q, t)=$ $\int d y \exp [i q y][\hat{\rho}(y, t)-\bar{\rho}]$, and made use of translationary invariance of the system. By inspection of Eq. (13) we find that the dominating contributions to the integral at large times come from values $q \ll a^{-1}$.

To evaluate $S(q, t)$ in the limit of small values of $q$, we make use of the Mori-Zwanzig projector operator formalism $[17,18]$. For this purpose we introduce the projection operators $\hat{P}=|\hat{\rho}(q, 0)\rangle_{\mathrm{eq}}\langle\hat{\rho}(q, 0)| /[N S(q, 0)]$ and $\hat{Q}=$ $1-\hat{P}$. Here, we make use of the Dirac notation and the brackets, \langle\rangle$_{\mathrm{eq}}$, indicate averaging with respect to Boltzmann weight. The time derivative of the dynamic structure factor can be written within this formalism as

$$
\frac{\partial S(q, t)}{\partial t}=-q^{2} D^{e}(q) S(q, t)+\int_{0}^{t} M\left(q, t-t^{\prime}\right) \frac{S\left(q, t^{\prime}\right)}{S(q, 0)} d t^{\prime}
$$

with an effective wave-vector dependent diffusion function, $D^{e}(q)=D_{S} H(q) / S(q)$, and the so-called memory function $M(q, t)=\left\langle\hat{\rho}(q, 0) \Omega_{B}\right| Q \exp \left[Q \Omega_{B} Q t\right] Q \times$ $\left|\Omega_{B} \hat{\rho}(q, 0)\right\rangle_{\mathrm{eq}}[13]$. Here, $H(q)=\left\langle\hat{\rho}(q, 0)\left|\Omega_{B}\right| \hat{\rho}(q, 0)\right\rangle_{\mathrm{eq}} /$ $\left(q^{2} D_{S} N\right)$ is the hydrodynamic function [19]. Detailed calculations show that $M(q, t)=\mathcal{O}\left(q^{4}\right)$ if HI is neglected, or taken into account in far-field approximation, $D_{i j}\left(y^{N}\right)=$ $D_{i j}\left(y_{i}-y_{j}\right)$ [13]. The full treatment of HI in the memory function gives contributions of order $q^{2}$, but the correction to $S(q, t)$ are within only a few percent, even for highly concentrated suspension with short-range interac- tions [20]. For the colloidal systems used in the experiments of Ref. [12], the far-field approximation of $D_{i j}\left(y^{N}\right)$ has been shown to be an excellent one [21].

For identical Brownian particles and HI neglected or treated on a pairwise additive level the solution of Eq. (14) for $S(q, t)$ is given by

$$
S(q, t)=S(q, 0) \exp \left[-q^{2} D^{e} t\right]+\mathcal{O}\left(q^{4} t\right),
$$

with $D^{e}=\lim _{q \rightarrow 0} D^{e}(q)$. Substituting Eq. (15) into Eq. (13), the second cumulant reads, in the asymptotic long-time regime,

$$
\kappa^{(2)}=4 \bar{\rho} S\left(\frac{D^{e} t}{\pi}\right)^{1 / 2}+o\left(t^{\epsilon / 2}\right) .
$$

Here, $S=S(0,0)$ is the relative compressibility [18] and the dependence of $\kappa^{(2)}$ on $x_{t}-x_{0}$ is of order $o\left(t^{\epsilon / 2}\right)$ due to the condition Eq. (8). The higher cumulants are given by $\kappa^{(2 n+1)}=0$ and $\kappa^{(2 n)}=\mathcal{O}\left(t^{1 / 2}\right)$ for $n \in\{1,2, \ldots\}$. In order to prove that the odd cumulants higher than one are exactly zero, we have made use of the backward operator to be Hermitian with respect to the Boltzmann weighted inner product, $\left\langle f \Omega_{B} g\right\rangle_{\mathrm{eq}}=\left\langle g \Omega_{B} f\right\rangle_{\mathrm{eq}}$, and invariance of the system with respect to translation and space inversion. The asymptotic scaling of the even cumulants can be found with the help of the mean-value theorem of integration and the fact that the correlation length between particles can be taken finite for physical systems in the fluid state. For a $2 n$-point correlation function one uses the mean-value theorem for $2 n-2$ integrals and then proceeds along the same line as for the second cumulant.

Using the explicit expressions for the cumulants we find from Eq. (11)

$$
\tilde{P}_{r}\left(\Delta x_{t}-\Delta z_{t}, t\right)=\frac{\bar{\rho}}{\left(2 \pi \kappa^{(2)}\right)^{1 / 2}} \exp \left[-\frac{\bar{\rho}^{2}\left(\Delta x_{t}-\Delta z_{t}\right)^{2}}{2 \kappa^{(2)}}\right] .
$$

From Eq. (6) we can derive finally an expression for the conditional PDF for $x(t)$,

$$
\tilde{P}\left(x_{t}, t \mid x_{0}, 0\right)=\frac{\bar{\rho}}{\left(\pi \kappa^{(2)}\right)^{1 / 2}} \exp \left[-\frac{\bar{\rho}^{2}\left(x_{t}-x_{0}\right)^{2}}{\kappa^{(2)}}\right] .
$$

Hence, the mean-squared displacement in the asymptotic 
limit is given by

$$
W(t)=\frac{S}{\bar{\rho}}\left(D^{e} t / \pi\right)^{1 / 2}+o\left(t^{\epsilon / 2}\right) \quad \text { for } t \gg \tau .
$$

The effective diffusion constant $D^{e}$ can be experimentally determined by a short-time measurement of $S(q, t)$ at $q \ll a^{-1}$ [22]. For times short enough that each individual particle does not "feel" the presence of the other particles by direct interactions, the integral over the memory function in Eq. (14) does not contribute, and thus there exists a unique relationship between $S(q, t)$ and $D^{e}$ as given in Eq. (15) with the second term on the right-hand side neglected. It is interesting to see that a long-time self-diffusion property like the MSQ can be determined to high accuracy from the short-time collective behavior of the system. This fact is a direct consequence of the single-filing condition imposed on our system.

Classical systems. - So far we have considered only interacting Brownian particles in the overdamped limit. For classical particle systems following Liouville dynamics, like atoms or molecules in the high temperature limit, we can essentially follow the same route as for the overdamped systems with the Liouville operator substituted for the Smoluchowsky operator. The analysis follows the same line as for overdamped systems with the result that the conditional PDF for classical systems is again Gaussian to leading order in time but normal diffusive,

$$
P\left(x_{t}, t \mid x_{0}, 0\right)=\left(4 \pi D_{s} t\right)^{-1 / 2} \exp \left[-\frac{\left(x_{t}-x_{0}\right)^{2}}{4 D_{s} t}\right],
$$

with self-diffusion constant $D_{s}=\overline{\boldsymbol{v}}(1-d \bar{\rho}) /(2 \bar{\rho})$ for hard-rod systems (cf. Refs. [4,5]) and $D_{s}=c_{s} S C_{V} /$ $\left(2 C_{P} \bar{\rho}\right)^{-1}$ for classical systems in the hydrodynamic regime [23]. Here, $\bar{v}$ is the average velocity of the particles, $d$ is the rod length, $c_{s}$ is the speed of sound, and $C_{V}$ and $C_{P}$ are the specific heats at constant volume and pressure, respectively. If the low-frequency sound modes are suppressed, like in the presence of a randomizing background (e.g., particle-wall interactions in a porous media), the MSD can be shown to scale like $W(t) \sim t^{1 / 2}$ [8]. This interesting property of single-filing systems will be the subject of a forthcoming paper.

In conclusion, we have derived a general theory for the asymptotic behavior of the MSD in single-filing systems. We have shown that $W(t)$ is determined by the short-time and large-scale collective behavior of the particles in the system. This relation holds true for any kind of interac- tion potential as long as the correlation length between the particles is finite, which is true for any physical system in the fluid state. Moreover, this relation is unique in the case of classical systems and in the case of overdamped systems with HI taken into account in far-field approximation.

We thank C. Bechinger, J. K. G. Dhont, and G. Nägele for helpful discussions.

*Corresponding author.

Email address: kollmann@lagash.dft.unipa.it

[1] T. E. Harris, J. Appl. Prob. 2, 323 (1965).

[2] A. Arratia, Ann. Probab. 11, 362 (1983).

[3] H. van Beijeren, K.W. Kehr, and R. Kutner, Phys. Rev. B 28, 5711 (1983).

[4] D. Jepsen, J. Math. Phys. (N.Y.) 6, 405 (1965).

[5] J. L. Lebowitz and J. K. Percus, Phys. Rev. 155, 122 (1967).

[6] K. Hahn and J. Kärger, J. Phys. A 28, 3061 (1995).

[7] H. van Beijeren, J. Stat. Phys. 63, 47 (1991).

[8] D. G. Levitt, Phys. Rev. A 8, 3050 (1973).

[9] S. Alexander and P. Pincus, Phys. Rev. B 18, 2011 (1978).

[10] V. Kukla et al., Science 272, 702 (1996).

[11] K. Hahn, J. Kärger, and V. Kukla, Phys. Rev. Lett. 76, 2762 (1996).

[12] Q.-H. Wei, C. Bechinger, and P. Leiderer, Science 287, 625 (2000).

[13] G. Nägele, Phys. Rep. 272, 215 (1996).

[14] D. L. Ermak and J. A. McCammon, J. Chem. Phys. 69, 1352 (1978).

[15] H. Risken, The Fokker-Planck Equation (Springer-Verlag, New York, 1989), 2nd ed.

[16] N.G.V. Kampen, Stochastical Processes in Physics and Chemistry (North-Holland Physics Publishing, Amsterdam, 1981).

[17] H. Mori, Prog. Theor. Phys. 33, 423 (1965).

[18] B. J. Berne and R. Pecora, Dynamic Light Scattering (Dover, New York, 1976).

[19] J. K. G. Dhont, An Introduction to Dynamics of Colloids (Elsevier, Amsterdam, 1996).

[20] P. Szymczak, Ph.D. thesis, Institute of Theoretical Physics, Warszawa, 2001.

[21] B. Rinn, K. Zahn, P. Maass, and G. Maret, Europhys. Lett. 46, 537 (1999).

[22] Clemens Bechinger et al. (to be published).

[23] Hard-rod systems are special in the sense that they do not "thermalize" locally. Thus it makes no sense to speak about a sound wave in such a system. 